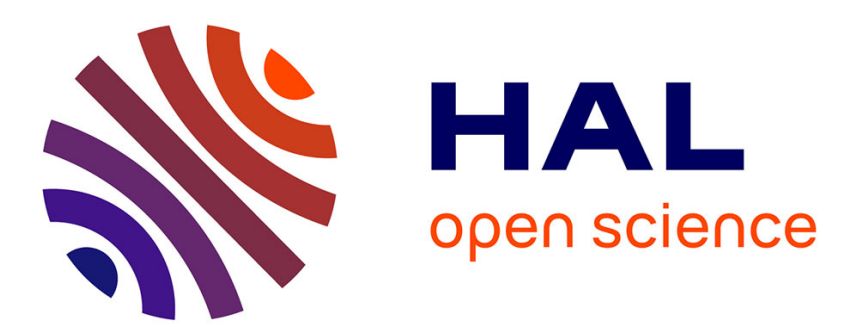

\title{
Evidentiel et progressif: quel statut grammatical pour la saillance prédicative?
}

Anaid Donabedian-Demopoulos

\section{To cite this version:}

Anaid Donabedian-Demopoulos. Evidentiel et progressif : quel statut grammatical pour la saillance prédicative?. Faits de langues, 2012, 39, pp.62-82. halshs-00722421

\section{HAL Id: halshs-00722421 https://shs.hal.science/halshs-00722421}

Submitted on 1 Aug 2012

HAL is a multi-disciplinary open access archive for the deposit and dissemination of scientific research documents, whether they are published or not. The documents may come from teaching and research institutions in France or abroad, or from public or private research centers.
L'archive ouverte pluridisciplinaire $\mathbf{H A L}$, est destinée au dépôt et à la diffusion de documents scientifiques de niveau recherche, publiés ou non, émanant des établissements d'enseignement et de recherche français ou étrangers, des laboratoires publics ou privés. 


\title{
Evidentiel et progressif : quel statut grammatical pour la saillance prédicative?
}

\author{
Anaïd Donabédian*
}

\section{INTRODUCTION}

L'objet de cet article est de contribuer à la réflexion sur la notion de saillance en tant que concept opérationnel en linguistique, notamment pour rendre compte de certaines catégories grammaticales dans les langues. Nous allons ainsi examiner la pertinence de la notion de saillance pour analyser les valeurs discursives, généralement non-discrètes, de deux TAM, l'évidentiel et le progressif, en arménien occidental (WMA). Cela nous conduira à interroger la notion de saillance elle-même pour déterminer le type de saillance concerné ici, mais aussi pour préciser son articulation avec les autres paramètres utilisés dans la description grammaticale, notamment en formulant des hypothèses sur la relation entre les valeurs dites prototypiques des tiroirs verbaux examinés (évidentialité, progressif) et les valeurs relevant de la saillance.

La notion de saillance est apparue en linguistique à propos des propriétés immanentes de certains référents, qui justifient dans certaines langues l'existence de stratégies syntaxiques fondées sur une hiérarchie entre référents argumentaux (constructions actancielles ou marquage différentiel de l'objet; rôle des propriétés référentielles dans la résolution des anaphores ou d'ambiguïtés référentielles). Elle concerne généralement des entités nominales, et met en jeu des propriétés intrinsèques, souvent scalaires, des référents (degré d'animéité, capacité de contrôle), ou des hiérarchies contextuelles (texte, situation), comme dans le fonctionnement de la deixis. C'est ce que F. Landragin (ici-même) qualifie de "saillance préalable », par opposition à la « saillance nouvelle ».

C'est à cette dernière que nous nous intéressons ici, même si nous préférons la qualifier de " construite », car il s'agit bien d'une opération qui hiérarchise les entités du discours dans le processus de l'énonciation. Le phénomène étudié en WMA en est un cas particulier, que nous appelons " saillance prédicative " car elle est caractérisée par la sélection de tiroirs verbaux marqués, l'évidentiel et le progressif (dans les cas, très nombreux, où elle ne correspond pas à un choix aspecto-modal contraint), et porte sur la prédication tout entière.

*SeDyL (UMR 8202), INALCO, CNRS, IRD ; adonabedian@inalco.fr 
L'extrême plasticité de la notion de saillance constitue à la fois un défi théorique, et tout l'intérêt de cette notion pour les faits grammaticaux comme celui que nous abordons ici. Landragin (2004) associe la saillance à ce qui est chargé d'émotion, ce qui détonne dans l'environnement, ou encore ce que le locuteur choisit de mettre en évidence. Ce versant de la notion, qui s'applique parfaitement à la saillance construite, peut sembler difficile à manier en grammaire, car peu opposable, et hautement relatif. De fait, ce que nous retenons ici de la notion de saillance pour la description grammaticale est justement cette propriété scalaire et relative, qui lui permet de rendre compte d'opérations de sélection grammaticale relevant de facteurs non discrets dans les langues. Cela nous conduira à interroger les notions de figure et fond qui sont en jeu dans les phénomènes observés, bien que leur instanciation ne soit pas toujours claire (ex. 13) et que leur rapport dépasse l'opposition binaire. Nous mettrons notamment cette notion en regard avec celle de 'non quelconque' (Milner 1978) que nous avons précédemment mise en œuvre pour rendre compte de ces phénomènes (Donabédian \& Montaut 2011).

Nous cherchons donc dans cet article à mettre en évidence le fonctionnement de la saillance en tant que phénomène multifactoriel, et à interroger la notion de saillance en tant que candidate au statut de catégorie grammaticale, puisque, comme nous le montrons dans l'article, elle est un excellent candidat pour justifier la sélection d'un tiroir verbal marqué. Pour ce faire, nous présenterons en 2. et 3. les deux tiroirs verbaux concernés par cette étude, avec leurs différentes valeurs et leur place dans le système, ce qui permettra de mettre en évidence leurs convergences énonciatives, puis nous confronterons en 4. l'ensemble de ces valeurs à la notion de saillance, avant de proposer notre conclusion en 5 .

Les catégories de l'évidentialité et du progressif sont grammaticalisées en arménien occidental : elles constituent un tiroir verbal avec un paradigme flexionnel propre. Dans les deux cas, on peut identifier des éléments référentiels fondant la valeur prototypique de la catégorie : la référence à une 'source du savoir' (ouï-dire, inférentiel) pour l'évidentiel, et la valeur aspectuelle de déroulement, de concomitance et de procès non borné pour le progressif. Mais dans les deux cas aussi, l'emploi de ces formes ne se réduit pas à un 'encodage' des notions prototypiques associées, notamment en discours, où les jugements de validité des énoncés sont difficiles à fonder sur des critères extralinguistiques référentiels (témoignage) ou contextuels (concordance des temps, etc.). Ainsi, dans de très nombreux exemples tirés de corpus spontanés, la commutation, respectivement avec un parfait ou un aoriste, ou avec un présent simple, donne des énoncés tout à fait grammaticaux (cf. infra ex. 4, 5, 8, 9, 10, etc.) sans qu'il soit nécessaire de manipuler le contexte même large. Cela explique d'ailleurs 
que l'évidentiel soit mal décrit dans les travaux normatifs, et que le progressif y soit tabouïsé1.

\section{L'EVIDENTIEL ${ }^{2}$}

L'évidentialité, introduite par les travaux de Chafe \& Nichols (1986), puis, en France, après G. Lazard (1956: 149), par les publications de Z. Guentcheva (1996) sous le nom de médiatif, et consacrée par l'étude extensive d'A. Aikhenvald (2004), occupe une place croissante en typologie. Après avoir été mise en évidence dans des langues où elle constitue une catégorie grammaticale spécifique (turc, albanais, bulgare, tibétain, langues amérindiennes, etc.), [on parlera alors de travaux sur les évidentiels] la notion typologique d'évidentialité a été utilisée comme étalon sémantique permettant d'étudier les différents modes d'expression de l'évidentialité dans les langues, $\mathrm{y}$ compris là où elle n'est pas grammaticalisée. Dans ce cas, la valeur retenue comme centrale est la source de l'information (ouï-dire) et le non-engagement (commitment) du locuteur [on parlera alors de travaux sur l'évidentialité].

L'évidentialité a aussi été abordée comme faisant partie du continuum des modalités (cf. Van der Auwera \& Plungian $1998: 85$ ), ce qui a fait progresser l'idée que la lecture évidentielle au sens littéral n'est pas la seule possible pour ces marqueurs. L'idée que l'expression de l'évidentialité a des corrélats modaux est apparue dès les premiers travaux sur les évidentiels, avec la mise en avant de la mirativité, d'abord exprimée comme 'sudden awareness' (Aksu Koç \& Slobin 1986). Dans de nombreuses langues, les valeurs de ces marqueurs sont, selon le contexte, le ouï-dire (source de l'information indirecte, non-commitment) et l'inférentiel (variante logique de la source de l'information), mais aussi le miratif (surprise, admiration), que DeLancey (1997) a proposé de dissocier de l'évidentialité. Un des défis de l'étude des évidentiels grammaticalisés, malgré le grand nombre de travaux qui leur sont consacrés, reste de comprendre ce qui fonde leur faisceau de valeurs, allant de la source de l'information (évidentialité prototypique), à la surprise et ses corrélats (sudden awareness, mirativité, $c f$. Montaut 2006), et aux autres valeurs modales de cette catégorie grammaticale.

La présente analyse s'appuie essentiellement sur l'arménien occidental, qui a un tiroir verbal évidentiel au passé, mais elle s'applique mutatis mutandis aux langues où les évidentiels sont issus de la spécialisation d'une forme de parfait (bulgare, albanais, turc). On remarque d'ailleurs que pour une bonne partie, les valeurs étudiées ici sont, dans des langues périphériques de l'aire, des valeurs

1 cf. Donabédian 2001b : le progressif n'est pas enseigné dans les grammaires scolaires, il est considéré comme populaire ou même dialectal, et dans la littérature il est presque exclusivement cantonné aux dialogues.

2 Nous désignons habituellement cette catégorie sous le terme de médiatif. Cependant, l'évidentiel est aujourd'hui une catégorie très débattue en typologie, et dans cet article, qui veut contribuer au débat général sur cette notion/catégorie, nous choisissons de conserver ce terme. 
contextuelles du parfait (arménien oriental, persan) ${ }^{3}$. La réflexion sur ce faisceau de valeurs et sur l'évidentialité dans l'aire Balkans/Anatolie est donc indissociable de la réflexion sur les modalités, comme l'a montré Johanson (2000) qui préfère le terme d'indirective pour cette catégorie.

Dans nos travaux précédents sur l'évidentiel en arménien occidental, nous avons mis l'accent sur une série de valeurs discursives étudiés dans des corpus spontanés ${ }^{4}$, montrant que les valeurs proprement évidentielles n'étaient pas majoritaires. L'analyse de ces exemples nous avait conduite à postuler (Bonnot, Donabédian et Seliverstova 1998, 1999; Donabédian 1999, 2001a) que ces valeurs relèvent d'un mode spécifique d'assertion, dans lequel le contenu propositionnel est asserté non pas en tant que tel, comme une information stabilisée, mais en tant qu'il contraste avec un autre point de vue, point de vue dont les différents types d'instanciation (état de conscience antérieur du locuteur, point de vue imputé à l'interlocuteur, doxa ou connaissance du monde) conditionnent le déploiement des différentes valeurs (surprise, polémique, glose). Cette notion de contraste est confirmée par le fait que ces énoncés sont presque obligatoirement sans segmentation thème/rhème, s'agissant d'une focalisation de l'énoncé complet (cf. Bonnot 2004), mais elle n'était pas tout à fait satisfaisante, comme dans le cas de l'apparition soudaine, qui amenait à postuler un contraste avec un état de fait neutre, ou dans le cas du ouï-dire, un contraste sur la valeur de vérité.

Nous voulons ici évaluer la valeur opérationnelle de la notion de saillance pour rendre compte de ces données, puis tenter d'expliquer ce que la mise en saillance discursive opérée par les évidentiels (alors miratifs ou modaux) dans le discours (prosodie, intonation, etc.) a en commun avec la core-value évidentielle (ouï-dire, mise en doute, non-commitment.

\subsection{Le marqueur dans le système de l'arménien occidental}

L'évidentiel en WMA est, comme le parfait, une forme verbale composée, mais où on trouve, au lieu du participe de résultatif d'origine adjectivale (sir-ac en, aimer-PFT AUX3PL, 'ils ont aimé'), une forme invariable en -er (sir-er en, aimer-EVID AUX3PL, 'ils ont aimé EVID'). Ainsi, alors qu'en arménien oriental, comme par exemple en persan, l'évidentiel apparaît comme une valeur contextuelle du parfait ${ }^{5}$, en WMA, parfait et évidentiel ont des formes distinctes en synchronie, même si la forme en -er est issue de l'ancien parfait de l'arménien classique.

${ }^{3}$ Ces valeurs peuvent s'ancrer dans d'autres tiroirs verbaux comme l'aoriste, excluant alors la valeur de ouï-dire (A. Montaut ici-même et Vassilaki \& Tsamadou (1995)), mais aussi à des niveaux totalement différents du système, comme nous l'avons montré avec C. Bonnot en comparant l'évidentiel arménien et l'accent de phrase non final en russe.

4 Notamment le corpus oral constitué par Kasparian (1992).

5 Nous ne suivons pas N. Kozintseva (1995), qui identifie un évidentiel en arménien oriental, dans la mesure où les valeurs évidentielles n'y sont pas associées à une marque propre, mais au parfait, dans certaines conditions de contexte et d'intonation. 
Aucune combinaison de personne ne rend l'emploi du médiatif agrammatical, contrairement à ce que l'on observe en coréen et en tibétain (Chang 1996; Tournadre 1996), En revanche, outre le fait que la catégorie n'existe qu'au passé accompli (comme en turc et en bulgare, mais pas en albanais où il concerne tout le système verbal), l'évidentiel n'est pas compatible avec le mode non-actualisé : ainsi, si pour toutes les autres formes analytiques du verbe, le paradigme oppose formes à auxiliaire au présent actualisé em et à auxiliaire non-actualisé ('subjonctif') ëllam, la forme en -er, elle, bloque cette opposition. Ainsi, seules les subordonnées complétives et circonstancielles (à l'indicatif) permettent un prédicat à l'évidentiel (ex. (19)), ce qui montre le caractère modal de la catégorie en arménien.

\subsection{Les valeurs sémantico-pragmatiques en arménien occidental}

La valeur prototypique de ouï-dire ou d'information non-constatée est observée dans la narration ${ }^{6}$ :

(1)

$$
\begin{aligned}
& \text { Maro-n amusnac'e!r } \bar{e} \\
& \text { Maro-ARTDEF marier-EVID AUX3SG } \\
& \text { 'Maro s'est mariée (paraît-il)!' }
\end{aligned}
$$

Bien qu'aucune source d'information ne soit identifiable, ces énoncés peuvent avoir un effet de polyphonie ou de non-engagement du locuteur. C'est cette valeur que les informateurs citent en priorité lorsqu'ils sont interrogés sur la forme en -er hors contexte. Mais d'autres valeurs, dont certaines sont difficilement associables à la non-constatation, sont observées en discours :

- Valeur dite d' inférence'

[Sortant de chez lui, le locuteur, qui n'avait pas remarqué qu'il avait plu, constate que le sol est mouillé]

$$
\begin{array}{lll}
\text { anjrew } & \text { ek-er } & \bar{e} \\
\text { pluie } & \text { venu-EVID AUX3SG } \\
\text { '(Tiens), il a plu! (pluie est venue)' }
\end{array}
$$

Le contexte suggère que l'assertion part du constat d'une trace de l'événement, et certains y voient une opération logique d'inférence. Elle peut être associée à une intonation exclamative ou non. Cela est cohérent avec le fait que l'événement est asserté au moment même de sa prise de conscience (sudden awareness). Dans le même contexte, la commutation avec le parfait est possible, mais elle indique que la notion de pluie était présente à l'esprit du locuteur (on attendait la pluie, elle était annoncée, on se demandait s'il allait pleuvoir, etc.).

${ }^{6}$ ARTDEF/ARTINDEF=article défini / indéfini, aux=auxiliaire, 1,2,3=personne (verbe) or degré (deixis), SG/PL=nombre, $\mathrm{EVID}=$ évidentiel, $\mathrm{PFT}=$ parfait, POSS=possessif, PROGR=progressif, $\mathrm{PRST}=$ présent, $\mathrm{AOR}=$ aoriste, $\mathrm{DEICT}=$ déictique, $\mathrm{PROH}=$ prohibitif. 
- Valeur de surprise (dite aussi 'mirative')

[Deux vieux amis se rencontrent. L'un est accompagné de sa fille, que l'autre n'a pas vue depuis des années]

$$
\begin{aligned}
& \text { ayji?k-d } \quad \bar{e} \quad m e c c^{\prime}-e ! r \quad \bar{e} \\
& \text { fille-POSS2SG est grandi-EVID AUX3SG } \\
& \text { 'C'est ta fille ? (Qu'est-ce qu')elle a grandi !' }
\end{aligned}
$$

Cette valeur, qui implique aussi un contexte de sudden awareness, semble exiger en outre une intonation exclamative. C'est le décalage entre l'attente du locuteur ('pas si grande') et ce qui s'impose à lui ('si grande') qui motive l'emploi de l'évidentiel. Ce type d'énoncé est souvent associé à un prédicat valorisant (admiratif). Mais il peut aussi être péjoratif (Gerc'er $\bar{e}$, 'Ce qu'elle a grossi !'). Il a aussi une forte affinité avec les prédicats gradables. Un parfait en - $a c$ exprimerait ici la confirmation d'une attente ('En effet, elle a grandi') et sa valeur pragmatique consisterait seulement à spécifier le contenu sémantique d'un prédicat déjà associé à ce sujet dans le contexte ('elle a changé').

\section{Un continuum de valeurs}

En contexte, la distinction entre ces trois valeurs n'est pas toujours claire, comme l'ont noté Aksu-Koç \& Slobin (1986) à propos de l'exemple turc Ahmed gelmiş, 'Ahmed est venu-EVID' qui peut selon les contextes aussi bien se gloser 'il est venu, on me l'a dit', 'je vois son manteau accroché, donc il est arrivé, ou encore 'je n'osais plus espérer qu'il vienne, mais il est venu'. De même, l'évidentiel a plusieurs valeurs simultanées dans ce dialogue de Chahnour?

(4) Do, Hrač', ësē nayim, as k'u ëracd kë vaylēe?

Alors, Hratch, est-ce que c'est des choses qui se font?

$\begin{array}{lllll}\text { norēe?n } & \check{c} \text { 'ocux } & \text { më } & \text { unec'-er } & \text { es } \\ \text { encore? } & \text { enfant } & \text { ART.IND } & \text { avoir-EVID } & \text { AUX2SG }\end{array}$

Tu as encore eu (EVID) un enfant ?'

Même si le contexte oriente ici vers le reproche ou l'ironie ('cela ne se fait pas'), il n'interdit pas l'interprétation de ouï-dire ('il paraît que '), d'inférence ('je vois que'), de surprise ('ça, alors !'), sans exclusive mutuelle.

\section{La valeur polémique, de glose, de commentaire}

Le rôle du contexte et la fluidité de ces valeurs suggèrent qu'elles n'épuisent pas la définition de la catégorie, et de plus, l'inventaire des valeurs n'est pas clos. La valeur de reproche, qui apparaît comme une valeur secondaire en (4), peut également être la valeur principale portée par l'évidentiel:

7 Pour les exemples extraits du corpus qui illustrent le fonctionnement sémantique en contexte, nous ne fournissons pas systématiquement la glose interlinéaire, mais nous indiquons dans la traduction le temps utilisé lorsqu'il n'y a pas de correspondance directe et univoque avec le temps français employé dans la traduction. 
[La belle-mère et la belle-fille cohabitent. Dans l'après-midi, la belle-fille dort. Une amie venue boire le café demande à la belle-mère où est sa belle-fille]

(5)a. paŕk-er $\overline{\mathrm{e}}$

couché-EVID est

'[figure toi qu'] elle s'est couchée !' (sous-entendu : quelle paresseuse !)

b. park k-ac $\bar{e}$

couché-PFT est

'Elle est couchée !' (pas de sous-entendu : c'est normal, elle est enceinte, ou malade)

Ainsi, le reproche ou la polémique, que la typologie ne répertorie pas comme des valeurs des évidentiels, s'ajoutent à l'inventaire des valeurs sémanticopragmatiques de la catégorie, ce que confirment les observations de Duchet et Përnaska (1996 : 40-41) sur l'albanais ('reprise polémique'), et de Meydan (1996 : 134) sur le turc ('des nuances exprimant le doute, la méfiance, l'ironie, la dénégation, l'indignation'). C'est dans la continuité de cette valeur que se situe celle de glose, de commentaire ou d'explication souvent véhiculée par cette forme (ex. 17, 18, 20, où l'on peu paraphraser l'évidentiel par 'tu vois?, hein ?').

\section{LE PROGRESSIF}

En tant que catégorie aspectuelle, le progressif est lié à l'inaccompli (présent ou imparfait), et vise le déroulement du procès sans considération de ses bornes. Cette valeur aspectuelle est, en arménien comme ailleurs, corrélée à des valeurs modales qui sont plus rarement décrites. Franckel (1989) a montré, à propos de 'être en train de' en français, comment la valeur aspectuelle et la valeur modale pouvaient s'expliquer par la même opération. Nous montrerons ici que valeurs aspectuelles et modales s'articulent de manière comparable en WMA.

\subsection{Le marqueur dans le système de l'arménien occidental}

WMA forme son présent à l'aide de la particule d'actualisation $k \ddot{e} / k$ ' $/ k u$, qui distingue sirem, "que j’aime" de kë sirem, "j'aime". Mais il existe un présent progressif, réputé dialectal et rejeté par la norme bien que très fréquent à l'oral, et formé par postposition de la particule $k o r^{8}$ au présent simple :

\begin{tabular}{|c|c|}
\hline $\begin{array}{ll}i ? n c \check{c} & \boldsymbol{k} \text { '-ën-es } \\
\text { quoi } & k \text {-faire-PRST2SG }\end{array}$ & $\begin{array}{l}\boldsymbol{k} \text { '-ašxatim } \\
\boldsymbol{k} \text {-travailler.PRST1SG }\end{array}$ \\
\hline 'Que fais-tu ?' & 'Je suis en train de $t$ \\
\hline
\end{tabular}

8 Prononcée [gor]. Les dialectes occidentaux recourent à des particules variées (cf. Gevorkian 1994). Gor, qui s'est fixé à Constantinople, a une affinité avec la particule d'actualisation WMA $k \ddot{e}$, et avec le suffixe progressif turc -yor, ce qui pourrait être un cas de convergence par contact. C'est à l'hypothèse populaire d'une origine turque, dans un contexte de 'tabou' de l'emprunt, que WMA doit l'idée que kor est dialectal. 
Outre les contraintes stylistiques (cf. note 9), le progressif, par rapport au présent simple (sans kor) exclut le sens de présent-futur (comparer (7)a et (7)b), et est peu naturel comme présent générique. Il suppose en effet un ancrage temporel concomitant à l'énonciation : $\begin{array}{llllll}\text { (7)a } & \text { hog } & m i & \ddot{e n e r} & \boldsymbol{k} \boldsymbol{u} & \text { gam [présent] } \\ & \text { souci } & \text { PROH } & \text { faire.2SG } & \boldsymbol{k} & \text { venir.PRST1SG }\end{array}$ 'Ne te fais pas de souci, je vais venir / je viendrai !'

Dans un tel contexte, le progressif bloque l'interprétation prospective :

(7)b hog mi ëner $k$ gam souci PROH faire.2SG $\boldsymbol{k}$ venir.PRST1SG PROGR 'Ne te fais pas de souci, je suis en train d'arriver !'

Avec certains verbes, l'emploi du progressif change l'interprétation sémantique du verbe, c'est un des rares cas où la substitution est impossible: comparer inč $\boldsymbol{k}$ '-ëllay [quoi $\boldsymbol{k}$-être.SUBJ3SG] 'Qu'est ce que cela peut faire ?' (statif, non-actuel), et inc' $\boldsymbol{k}$ '-ëllay kor [quoi $\boldsymbol{k}$-être.SUBJ3SG PROGR] 'Qu'est ce qui se passe ?' (dynamique, actuel).

Traditionnellement, l'interprétation de kor est donc :

- en linguistique : purement aspectuelle (progressif)

- en grammaire normative: purement stylistique (non standard, non pertinent)

Nous avons abordé le second point dans (Donabédian 2001b). Quant à l'interprétation purement aspectuelle de 'procès en cours', plusieurs indices montrent qu'elle n'épuise pas la description du marqueur.

\subsection{Les valeurs non-aspectuelles du progressif}

En WMA, le présent en kor n'est pas incompatible avec les verbes statifs :

[Une jeune domestique se plaint des assiduités du jeune homme de la maison auprès de l'intermédiaire qui l'a placée. Celle-ci lui rétorque : ]

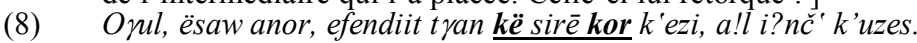

'Ma fille, lui dit-elle, [mais c'est que] le fils de ton effendi t'aime-PROGR, que veux tu de plus?' (Zohrab, Postal)

Le progressif souligne ici la valeur interprétative de l'assertion, qui vise à réfuter le point de vue de la jeune femme qui se sent agressée.

On trouve également des exemples avec des verbes comme 'vouloir', 'savoir' (17), 'avoir honte', 'se souvenir' (10) (fréquemment à la forme négative), et même au présent générique (18), autant d'éléments incompatibles avec le progressif au sens aspecto-temporel (déroulement du procès et concomitance 
temporelle). On retrouve dans les valeurs non-aspectuelles du progressif des valeurs déjà observées pour l'évidentiel :

- $\quad$ Reproche, indignation :

(9) Bayc dun arek ajusteur èir, inču gorc čënes? čes p'ntŕer kor. inč u? inć 'u ink'zink'd jger es? (Chahnour)

Mais toi, tu étais un bon ajusteur. Pourquoi tu ne travaillerais pas? tu ne cherches PROGR [même] pas. Pourquoi? Pourquoi tu te laisses aller [tu t'es abandonné-EVID] [comme ça]?

- Détrimental, regret :

(10) čem yišer kor

NEG.PRST1SG Se.souvenir PROGR

Je ne m'en souviens pas,

Kostan, me $k^{\prime}{ }^{\prime}$ 'ir inci, es im Hayr Merë čem yišer kor.

Kostan, aie pitié de moi, je ne me souviens pas PROGR de mon Notre-Père. (Chahnour)

Dans le roman, le locuteur réagit ici à l'attente de l'interlocuteur, mais il pourrait s'agir d'un contexte où il tente de se souvenir mais n'y parvient pas (glosable par un prédicat négatif : «vraiment pas ! j'ai beau faire! »).

- Argumentation, polémique (voir aussi l'exemple 8 ci-dessus):

(11) O $\check{c}$ ', čunim, k'ësem kor, anpayman k'uzes ëllay, čunim!

'Non, j'en ai pas, je te dis PROGR, tu veux absolument qu'il y en ait, j'en ai pas !' (Kasparian 1/57)

[un visiteur insiste pour voir le personnage bien qu'on lui ait dit qu'il était absent]

(12) Ohoo!!!, irar čenk' hasknar kor... bayc' sireli parons, jezi k'ësem or an hima meknec 'aw pandogēn...

'Ohoo, mais [on dirait que] nous ne nous comprenons PROGR pas ... mais mon cher Monsieur, [puisque] je vous dis qu'il vient de quitter l'hôtel...' (Chahnour)

- Tension dramatique :

[C. raconte ses vacances d'été sur les îles du Bosphore, au présent d'habitude, puis]

(13) As amaŕ, amen amaŕuan pēs Knalë gac'ink'. Amen ban law k'anc'nēer kor. Aŕtunerë uš k'ellayink' kor. Amboŷj ôrë awazanë k'anc'ënēink' kor. Gišernerë k'ič më ënkernerov durs k'ellayink' kor, šat anuor èr payc'... amen mard gitē or erkrašaržë e raw... (puis récit à l'aoriste)

'Cet été, comme tous les étés, nous sommes allés (AOR) à Knale. Tout se passait PROGR bien. Les matins, on se levait PROGR tard. On passait PROGR toute la journée à la piscine. Les soirs, on sortait PROGR un peu avec des amis, c'était vraiment bien, mais... tout le monde sait qu'il y a eu (AOR) le 
tremblement de terre...' (recueilli par F. Deyirmendjian, dossier de maîtrise).

On le voit, ces différentes étiquettes sont conventionnelles, elles ressortissent en fait de mécanismes similaires : dans tous les cas, deux représentations s'opposent, que ce soit celle du locuteur et de l'interlocuteur, celle qui est activée chez le locuteur et ce qui s'impose à sa conscience au moment de l'énonciation, ou encore celle qu'on pourrait considérer comme allant de soi et une réalité 'non quelconque' qui s'impose à la conscience. En cela, l'opération énonciative qui sous-tend la sélection de ce marqueur est très proche de celle qui correspond au choix de l'évidentiel pour un procès accompli.

Ces valeurs modales font écho à celles que décrit et analyse Jean-Jacques Franckel (1989) à propos de « en train de » en français, même si les emplois de kor en arménien ne sont pas totalement superposables (cf. ex. 8, 9, 10, 16, 17, 18 , comportant des prédicats statifs, génériques ou négatifs, qui même avec des périphrases, ne se laissent pas traduire par « en train de » en français).

Franckel montre que les différentes valeurs du progressif français, y compris les valeurs modales, s'expliquent par le fait que le contenu propositionnel P est bien actualisé, mais que son contenu notionnel (au sens du domaine notionnel chez Culioli, structuré en Intérieur I et Extérieur E) n’est pas stabilisé (la « bonne » valeur I et les autres valeurs E contenues dans le domaine notionnel sont encore en concurrence dans cet ancrage temporel, I étant activé mais pas totalement actualisé). Cette analyse lui permet d'extraire l'invariant qui unit la valeur aspectuelle du progressif et ses valeurs modales en français. Il met en évidence deux familles de valeurs, les valeurs téléonomiques, où I est visé par le procès (valeur aspectuelle de procès en cours, objectif à atteindre, intentionnalité), et les valeurs antitéléonomiques, où la localisation de $\mathrm{P}$ est première par rapport à l'activation de la valeur notionnelle (il y a quelque chose, et l'énoncé consiste à lui attribuer la «bonne valeur » en termes notionnels, à le qualifier : valeurs de prise de conscience, mise en garde, rejet ou démarcation, appréciation négative avec les verbes d'état).

La coexistence de valeurs conatives, intentionnelles (1989: 70) : On est en train de tout déménager ; (1989: 71): il est en train d'enfoncer la porte) avec les valeurs d'effacement de l'intentionnalité (Regarde! Les lilas sont en train de fleurir !), d'anti-téléonomie (Il y a un papier qui est en train de tomber !), de rejet (je suis en train de travailler!) ou d'appréciation négative (Il est en train d'être méchant), et l'analyse en termes de discordance, de décalage entre le perçu et le représenté avec la dimension d'évaluation qui accompagne la bifurcation vers I et E dans l'analyse de Franckel (1989: 65-66), concordent totalement avec les valeurs relevées en WMA non seulement pour le progressif, mais aussi pour l'évidentiel. 
Comme nous l'avons montré, les emplois discursifs (hors valeurs de ouï-dire et de concomitance temporelle) de l'évidentiel et du progressif ont de nombreuses affinités : l'éventail de leurs valeurs est comparable, ces valeurs sont en outre hautement variables en fonction de plusieurs paramètres (prosodie, configuration énonciative, contexte), et gradables en termes d'intensité.

Ainsi, les deux tiroirs verbaux apparaissent bien comme des formes marquées, qui s'inscrivent dans un choix paradigmatique binaire. Cependant, du fait de leur affinité avec la modalité, ce choix n'est pas nécessairement corrélé à des contraintes de grammaticalité : la commutation d'une forme marquée avec une forme non marquée n'est jamais agrammaticale, et l'emploi d'une forme marquée là où la forme non marquée est attendue pourra soit intriguer l'interlocuteur sur les intentions du locuteur (et susciter une réaction comme 'Qu'est-ce que tu sous-entends', ce qui indique que la valeur potentielle de la forme marquée reste activée même si elle n'est pas immédiatement interprétable), soit être perçu comme stylistiquement marqué (non-canonique, dialectal, populaire). On voit donc que le choix grammatical qui fonde les couples présent/progressif et parfait/évidentiel, malgré son caractère paradigmatique et binaire, n'en a pas moins un caractère subjectif, renforcé par le marquage prosodique (emphase) auquel il est fréquemment associé.

Les propriétés sémantiques de ces catégories, elles aussi, malgré le trait de bipolarité souligné plus haut, ne peuvent pas être réduites à une structure binaire ou de contraste, et en cela, elles rejoignent la configuration de la notion de saillance :

1) en termes qualitatifs (contenu notionnel, Qlt), il y a bien dans nos énoncés marqués une discordance entre deux représentations, qui ne sont pas dans une relation symétrique, tout comme l'opposition figure $\sim$ fond qui fonde la notion de saillance. La valeur assertée est présentée comme 'distinguée', ce qui l'oppose, non pas binairement à une valeur de même ordre qu'elle, mais potentiellement à toute autre valeur, c'est-à-dire à un continuum de valeurs. Dans le cas des prédicats gradables, ou de l'expression du haut degré, cela est particulièrement clair, mais pour les événements inattendus ponctuels, on peut gloser les énoncés marquée par 'cela et rien d'autre', 'cela et bien cela', ou encore tout simplement 'vraiment cela', ce qui reflète la structuration non binaire du domaine notionnel.

2) en termes quantitatifs (actualisation de la relation prédicative, Qnt - cela a vraiment eu lieu), on identifie l'effet de sudden awareness, de surprise, de contre-expectation, qui correspond à l'effet de pop-up décrit pour la saillance' Là encore, la relation figure fond n'est pas dans une stricte opposition binaire : 'cela a vraiment eu lieu' construit la figure, mais le fond n'est pas construit, ce qui explique la limite de l'analyse de ces marqueurs en termes de contraste, ce dernier supposant une symétrie entre deux valeurs. En d'autres termes, au plan

${ }^{9}$ Même si l'analogie ne peut pas être totale : dans certains cas, comme l'interprétation ou la polémique, il n'y a pas de véritable irruption soudaine à la conscience, le contenu propositionnel est déjà activé chez le locuteur, mais quelque chose du contexte (par ex. s'il attribue à l'interlocuteur un point de vue différent) le pousse à le réactiver. 
Qlt comme au plan Qnt, le contraste n'est qu'un cas particulier d'opposition entre figure et fond, où ce dernier est également construit dans le contexte.

3) en termes de hiérarchie communicative, ces marqueurs sont, en discours, associés à un focus intonatif (saillance physique), et la non-segmentation Thème/Rhème qui correspond à la focalisation de la relation prédicative dans son ensemble (Bonnot 2004), ce qui fait que ces énoncés ressortissent de la classe des énoncés thétiques marqués, à valeur informative faible sinon nulle, d'où leur affinité avec le commentaire, la glose, la caractérisation d'une situation.

$\mathrm{La}$ focalisation en arménien est marquée prosodiquement par une augmentation de l'intensité et une intonation montante-descendante, ce qui implique en termes syntaxiques que le constituant focalisé ne soit pas en position finale dans l'énoncé, puisque l'arménien est une langue à accent tonique final. Ainsi, si le constituant focalisé est un complément, il sera placé juste avant le verbe (l'arménien a un ordre de base à verbe final), mais s'il est en position finale, il a besoin d'un clitique pour que la chute intonative soit possible. Pour l'évidentiel, comme pout toute forme analytique, c'est l'auxiliaire qui joue ce rôle, pour le progressif, il n'est pas anodin que la particule choisie soit postposée, car c'est elle qui jouera ce rôle. Sémantiquement, cette focalisation a une valeur de focus de contraste (binaire) ou d'emphase (non binaire, 'distingué'). Dans le cas d'un focus sur un argument, il est traduit par une clivée en français ('c'est lui qui/que'). Dans le cas d'une focalisation de la prédication, comme celle qui se présente dans la majorité des énoncés traités ici, la valeur de la focalisation converge avec celles des marqueurs étudiés ici. De fait, la focalisation entraîne l'emploi de ces formes, au présent, pour des raisons d'accent tonique, au passé pour distinguer l'emphase sur le résultat (parfait) et l'emphase sur l'événement (évidentiel).

Les exemples ci-dessous montrent que les marqueurs de WMA examinés ici sont des opérateurs de saillance, qui sont souvent renforcés par des marques de focalisation. Ils promeuvent la relation prédicative qu'ils marquent au statut de 'figure', distinguée par rapport à un fond qui ne l'est pas. La diversité des valeurs en contexte, et même la fluidité des valeurs pour un même contexte, est ainsi imputable à l'articulation entre grammaire et pragmatique : le choix du TAM marqué présente la relation prédicative comme non quelconque ('c'est ça et bien ça', 'c'est vraiment ça' ou c'est vraiment arrivé'), et les valeurs se déclinent en fonction de l'intention pragmatique du locuteur au moment de ce choix, ce qui suggère des gloses comme 'tu n'a pas l'air de le croire, mais...'; 'ce n'était pas comme cela avant'; 'cela ne devrait pas être comme cela'; 'je n'en crois pas mes yeux', 'je trouve cela intolérable', 'j'ai beau faire', etc., qui, comme on le voit dans les exemples cidessous, en discours, mais également en récit, se déclinent à l'infini.

\subsection{Emplois en discours}

De nombreux exemples correspondent à une focalisation de l'énoncé au complet, avec, au plan prédicatif, une valeur de pop-up (sudden awareness ou surprise en 16), et, au plan notionnel, une bipolarité qui se manifeste par les valeurs d'effacement de l'intentionnalité $(14,15,18)$ ou d'antitéléonomie (avec les valeurs détrimentales en 
16, 17). On remarque dans ces exemples de nombreux prédicats négatifs

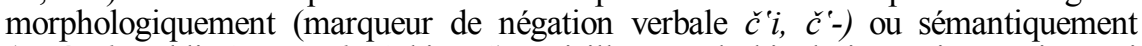
(mornal 'oublier', awruil 's'abimer'), qui illustrent la bipolarité en jeu, mais aussi des prédicats gradables (18) pour lesquels le focus porte sur le haut degré. Dans tous ces exemples, le prédicat est focalisé, ce qui se manifeste par une intonation montante descendante (par exemple pour 14, moŕc 'e $\boldsymbol{T} \mathbf{r}$ ei $\mathbf{y}$ ) et une augmentation de l'intensité, mais également par l'impossibilité d'une pause entre groupe nominal et groupe verbal, soit que le groupe nominal ne soit pas exprimé (cf. 17, 18), soit qu'il soit postposé au verbe (14). En (16), où la structure phrastique permettrait cette segmentation, on voit que l'emploi de l'évidentiel la bloque.

[en cherchant des outils pour réparer la bicyclette, S. regarde dans les affaires de son ami. Soudain, elle trouve un petit sac, qu'elle observe et commente]

(14) Hmm, as anušik é, mórć 'er ēi ays payusakd

'Hmm, ça, c'est mignon, j'avais oublié-EVID que tu avais un sac comme ça !'

[trois étudiants réparent une bicyclette. Après un temps de silence et de manipulation, A., qui dirige les travaux, dit, un peu agacé, à B. qui a entrepris de tenir la bicyclette immobile pour l'aider: ]

(15) B. - Voilà, voilà atika vaŕec 'aw 'Voilà, voilà, celle-là s'est allumée-AOR.'

A. - Vaŕi kor?

B. - Ha!, ha!, vare é

'Elle est allumée [elle brille-PROGR.]?'

C. - Etewinë várer

'Oui, oui, elle s'est allumée-EVID!'

(16) - As čci vaŕir kor hima al'

'Celle de l'arrière s'est allumée-EVID !'

'Celle-là, elle s'allume PROGR plus, maintenant.'

As inč 'u, amen inč' tern é, inč ‘u? čc $i$ varir

'Mais pourquoi, tout est bon! pourquoi elle s'allume pas?'

- Lampë ov gité awrue?r $\bar{e}$

'C'est peut-être l'ampoule qui est grillée ?'

[ampoule-ARTDEF qui sait abîmé-EVID AUX3SG]

(*-Lampë // ov gitē awrue?r $\bar{e}$ )

Dans certains cas, à la première personne, l'effet de pop-up est lié non à la surprise, mais à un état interne dont l'intensité est soulignée, et qui acquiert une dimension de commentaire sur la situation observée ('je ne sais pas, bon sang'), la valeur sémantique étant toujours l’antitéléonomie, le détrimental.

[au téléphone, programmation d'une soirée et des points de rendez-vous. A. parle au téléphone, et B. l'interroge pour suivre le déroulement des choses.]

(17) A. - Allo, oui ...

B. C'est P.?

A. Oui, oui

B. Qu'est-ce qu'il dit?

A. C'em giter kor, R-in č'enk' krnar kor gtnel, amen aten occupé è, kam al débranché ërer en

'J'en sais PROGR rien, on arrive PROGR pas à trouver R., c'est toujours occupé, ou alors ils ont débranché-EVID (...)' 
[S. se prépare à couper les cheveux de A. A. montre qu'il a récemment coupé une mèche lui-même car les cheveux avaient beaucoup poussé depuis la dernière coupe]

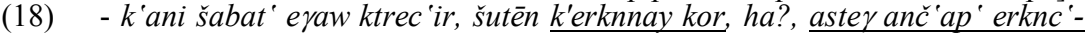
er $\bar{e} r$, tesar $i$ ? nč ëri, $c^{\prime} i$ tesar hos?

[là tellement grandir-EVID aux-past3sg]

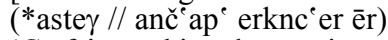

'Ca fait combien de semaines que tu me les as coupés, ça pousse (PROGR.) vite, hein, ça avait tellement poussé-EVID, tu as vu ce que j'ai fait, tu as pas vu, là ?'

\subsection{Emplois de type récit}

En récit, la valeur d'effacement de l'intentionnalité se traduit souvent par la combinaison d'un effet de sudden awareness et d'une valeur de contreexpectative associée au contenu propositionnel (d'où une incrédulité).

(19) K’ičer miayn nšec 'in, or geji eresēn inkac 18 tarekan lakotë, sewta è kaper Srapenc" pztik harsin ew yačax aybiwri čamban nazanin anc 'nelun kë spasēr.

'Peu de gens remarquèrent que le jeune vaurien de 18 ans dédaigné par le village, était tombé-EVID amoureux de la petite bru Srapian, et que souvent il attendait la belle sur le chemin de la source.' (Hamastegh, Premier amour)

Dans ce récit, on constate ici une configuration marquée à plusieurs égards : l'ordre auxiliaire-participe (sewta ē kap-er 'amour AUX lier-EVID' au lieu de l'ordre standard sewta kaper $\bar{e}$ ) place l'objet sewda 'amour' en position focalisée avant l'auxiliaire ${ }^{10}$, et par ailleurs on observe ici une exceptionnelle segmentation thème/rhème (marquée par la virgule) habituellement bloquée par l'évidentiel. Il s'agit de la configuration décrite par C. Bonnot (1997:29 et 2004:264) pour l'exemple Vanja-to / mašin-u kupi-l, [Vanja-to voiture-acc acheter-perf3sg ; 'Vania, il a achété une voiture !], dans laquelle le contraste sur le thème (qui suppose a priori que l'association de ce thème à Vanja était hautement improbable) entraîne la possibilité d'une segmentation thème/rhème malgré le focus rhématique.

L'énoncé suivant se situe à la frontière entre récit et discours, du fait de la dimension d'analyse, de commentaire, associée au contenu propositionnel : le focus porte sur le rhème au complet, qui qualifie la situation.

[Un couple paresse au lit. Enoncé isolé : elle réfléchit à voix haute]

(20) Elle : As fatalité-n mezi erkuk'nis iraru kaper è xnday kor vranis 'Cette fatalité, elle nous a liés-EVID tous les deux, et elle se moque-Progr de nous.'

où progressif et évidentiel sont mis en série sans joncteur ('cette fatalité, elle nous a liés-EVID se moque-PROGR de nous') .

Mais en récit aussi, le cas particulier du contraste binaire n'est pas exclu, comme le montrent (21) et (22), sémantiquement apparentés à la négation :

${ }^{10}$ Ce procédé est très répandu et bien décrit en arménien oriental, il est plus rare en arménien occidental. 
[S. raconte comment elle est rentrée en vélo un jour d'extrême fatigue. Par amour propre, elle n'a pas voulu descendre, mais tout près de la maison, dans une côte]

(21) Otk'ers č em zgar kor, velo-n minakë kë k'ale kor, quoi, au ralenti, $1 \mathrm{~km} / \mathrm{h}$, ov gitē asank, ouin, kamac' kamac' asank, mérer ēi, verjēen Parc Montsouris hasay nē ësi uzacnin ësen, k'î̀nam.

'Je sens-PROGR. plus mes pieds, le vélo roule-PROGR. tout seul, quoi, au ralenti, $1 \mathrm{~km} / \mathrm{h}$, quelque chose comme ça, ouin, tout doucement comme ça, j'étais morte-EVID., après, en arrivant Parc Montsouris, j'ai dit: ils disent ce qu'ils veulent, je descends.'

Le vélo roulant 'tout seul' peut être paraphrasé par un prédicat négatif : 'sans moi'.

[Discussion autour d'un verre. B. va en Italie, et propose à A. de l'accompagner]

(22) A. - 'Moi, moi, jusqu'à ce que j'aie mes papiers, dans ma situation, je sors pas du pays, pour aller dix fois courir après des visas à la dernière minute, ouf !' (en français dans le corpus)

B. - Amen frontière-nerë haner en, douane-in, drami hamar ewrobayi

[Toutes frontières enlevé-EVID AUX3PL.]

'Ils ont enlevé toutes les frontières à la douane, pour la monnaie européenne...'

A. - I? nč haner en 'Qu'est-ce qu'ils ont enlevé-EVID ?'

Dans ce contexte argumentatif, 'ils ont enlevé toutes les frontières' s'oppose à l'opinion de l'interlocuteur qui pense qu'il devra faire des demandes de visas (la pause entre thème et rhème est impossible (*Amen frontière-nerë // haner en).

\section{CONCLUSIONS}

Les propriétés que les deux marqueurs grammaticaux de TAM présentés ici confèrent aux énoncés dans lesquels ils apparaissent relèvent de plusieurs domaines d'analyse : TAM, pragmatique, prosodie, hiérarchie communicative, avec une forte dimension exclamative. Cela explique que ces marqueurs résistent aux analyses qui abordent les valeurs modales en termes paradigmatiques binaires (valeur de vérité oui/non, possible oui/non, nécessaire oui/non), et à celles qui opposent sémantique (valeur obligatoire, non dépendante de l'interlocution) et pragmatique.

Nous avons montré ici à travers les propriétés contextuelles de ces marqueurs, ainsi que certaines propriétés formelles des énoncés où ils apparaissent (nonsegmentation thème/rhème) qu'ils ont pour effet une mise en saillance de la relation prédicative, qui est ainsi présentée comme distinguée ou non quelconque du point de vue du locuteur, de l'interlocuteur, ou de la doxa.

Cette affinité de la notion de saillance avec les notions évoquées dans notre analyse (focus, thétique, rhématique, contraste) est soulignée dans de nombreux travaux. Les analogies avec la saillance perceptive (visuelle), la notion de cadre, de positionnement par rapport au cadre, d'isolement de la figure sur un fond, correspondent également aux configurations discursives évoquées ici, et font d'ailleurs écho aux notions élaborées ailleurs de windowing of attention, 
foregrounding et backgrounding (Langacker 1991, 1999, Talmy 1978, 2000). Nous avons souligné en 4. que le caractère asymétrique du rapport entre figure et forme, non réductible à un simple contraste de terme à terme, était essentiel pour la notion de saillance et son application aux catégories étudiées ici. Cela est corrélé au caractère non discret des mécanismes en jeu. Ce qui rend les catégories étudiées non discrètes est que soit le prédicat est gradable et la valeur de commentaire porte sur le haut degré (ex. 3 et 18), soit elle porte sur l'actualisation même de ce procès précis, ce qui consiste dans les deux cas en un commentaire sur le domaine notionnel concerné ('cela, une chose pareille, a vraiment eu lieu'). Ainsi, même lorsque les points de vue s'opposent, nous n'avons pas affaire à une simple négation, qui opérerait sur l'actualisation du contenu propositionnel (Qnt), mais à une opération sur le domaine notionnel (Qlt) comme dans l'exemple cité dans ce volume par F. Landragin (ex. 1) « (...) un téléphone pour té-lé-pho-ner ». Ici, il s'agit de souligner une valeur centrée sur l'attracteur «téléphoner et rien d'autre, purement téléphoner », qui est propice à la valeur de glose; de même dans l'exemple 2 de F. L., on entend « c'est justement à lui et à personne d'autre que je pensais ».

Ainsi, on voit que les marqueurs de TAM considérés ne portent pas (ou pas seulement, dans le cas du progressif) sur les bornes du procès (aspect), et sachant que leur valeur spécifique s'oppose respectivement à celle des formes non marquées de présent simple et de passé accompli (temps), il semble qu'elles relèvent de la modalité. Il ne s'agit cependant pas d'une modalité portant sur la valeur de vérité, le possible, le nécessaire (modalité paradigmatique au sens de Van der Auwera et Plungian 1998 : 80), mais sur un focus assertif, qui implique la dimension de prise en charge ou de commitment de l'énonciateur, que l'on peut gloser par c'est vraiment cela qui est le fait. C'est dans ce vraiment que réside la discordance évoquée par Franckel au sein du domaine notionnel I/E qui n'est, elle aussi, pas paradigmatique. Van der Auwera et Plungian (1998), dans leur proposition de carte sémantique des modalités, déclarent se limiter pour l'heure à la modalité épistémique ainsi qu'à l'inférentialité en tant qu'elle est liée à l'épistémique (l'emploi inférentiel de type «il doit avoir plu »), qui pour eux doit absolument tenir compte du paramètre speakers commitment, laissant hors du champ les autres valeurs des évidentiels. Ils intègrent donc la dimension de prise en charge énonciative dans le champ des modalités, mais pas l'évidentialité, en suggérant qu'elle pourrait relever plutôt d'un type illocutoire (illucutionary type de la grammaire fonctionnelle).

Nous avons cherché à montrer que les emplois discursifs de l'évidentiel et du progressif en arménien occidental relèvent de ce que nous avons appelé la saillance prédicative, qui se manifeste dans ces contextes à la fois au plan formel (focus prosodique et rhématisation de la prédication) et au plan notionnel (le contenu notionnel étant présenté comme distingué, et l'énoncé permettant de déployer une série de valeurs résultant d'une discordance entre une 'bonne valeur' et les autres). La saillance prédicative, ainsi mise en œuvre en tant que catégorie grammaticale se caractérise par son caractère non discret et subjectif (elle est une manifestation du speakers commitment non pas nécessairement pour ce qui est de la valeur de vérité de l'énoncé, mais pour ce qui est du domaine notionnel de la prédication). Nous avons vu que la notion de saillance prédicative permet en outre de saisir un grand nombre de propriétés de la syntaxe de ces marqueurs : comme l'aspect, elle est une 
catégorie de l'énoncé et non du verbe, comme les modalités, elle est non discrète et référentiellement non contrainte, et, elle est multi-vecteur (cf. la cooccurrence de la saillance physique avec la prosodie). L'étape ultérieure de ce travail pourrait être de tester l'hypothèse selon laquelle les phénomènes décrits ici fonderaient en réalité un type illocutoire particulier, ce qui renouvellerait le champ de la réflexion sur l'évidentialité en typologie linguistique, mais pourrait également donner un éclairage nouveau sur la saillance prédicative, en tant que configuration particulière de saillance construite.

\section{REFERENCES BIBLIOGRAPHIQUES}

Aikhenvald A. Y., 2004, Evidentiality, New York, Oxford University Press.

Aksu-Koç A. \&, Slobin D., 1986, A Psychological Account of the Development and Use of Evidentiels in Turkish, in Chafe \& Nichols (eds), Evidentiality: The Linguistic Coding of Epistemology, Norwood, Abex Publ., p.159-167.

Bonnot C., 1997, Pour une definition formelle et fonctionnelle de la notion de thème (sur l'exemple du russe moderne, Guimier C., (ed.), La thématisation dans les langues, Berne, Peter Lang, p. 15-31.

Bonnot C., 2004, Relation préconstruite et focalisation : pour une analyse unitaire des énoncés à accent non final en russe moderne, Slovo 30/31, p. 211-270.

Bonnot C. \& Donabédian A., 1999, Lorsque la morphosyntaxe rencontre la prosodie: accent non final en russe et médiatif en arménien, Faits de Langues, 10, p. 182-190.

Bonnot C., Donabédian A. \& Seliverstova O., 1998, Enoncés à accent initial en russe et énoncés au médiatif en arménien occidental: une convergence fortuite?, Proceedings of the $16^{\text {th }}$ International Congress of Linguists, Pergamon, Oxford, paper $\mathrm{n}^{\circ} 0323,18$ pages (CD-ROM).

Chafe W. \& Nichols J. (eds), 1986, Evidentiality: The Linguistic Coding of Epistemology, Norwood, Abex Publ.

Chang I., 1996, Représentation médiatif d'un suffixe verbal, -teo, en coréen contemporain, in Guentchéva (ed.), L'énonciation médiatisée, Paris, l'Information grammaticale, Louvain, Peeters, p. 183-194.

DeLancey S., 1997, Mirativity: The grammatical marking of unexpected information, Linguistic Typology I, p. 33-52.

Donabédian A., 1996, Pour une interprétation des différentes valeurs du médiatif en arménien occidental, in Guentchéva (ed.), L'énonciation médiatisée, Paris, l'Information grammaticale, Louvain, Peeters, p. 87-108

Donabédian A., 1998, Mode d'expression de l'accompli et aspectualité en arménien occidental, Actances 9, p. 13-32.

Donabédian A., 1999, Négation analytique et médiatif en arménien occidental: homonymie ou lien systémique?, Cahiers de Linguistique de l'INALCO 1, p. 23-41.

Donabédian A., 2001a, Toward a Semasiological account of Evidentials: an Enounciative Approach of -er in Modern Western Armenian, Journal of Pragmatics 33/3, p. 421-442.

Donabédian A., 2001b, Tabou linguistique en arménien occidental : 'gor' progressif est-il 'turc'?, in A. Donabédian (ed.), Langues de diaspora, langues en contact, Faits de Langues 18, p. 201-210. 
Donabédian A., 2002, Médiatif et progressif en arménien occidental : convergences discursives, in Donabédian A. \& Ouzounian, A. (eds), Actes du VIème Colloque International de Linguistique arménienne, Paris, INALCO, Slovo 24-25, p. 343-357

Donabédian A. \& Montaut A., 2011, Saillance syntaxique et énonciative en hindi et en arménien : vers une définition de la saillance linguistique, in Inkova O. (ed.), Saillance, aspects linguistiques et communicatifs de la mise en évidence dans un texte, Vol. 1, Annales littéraires de l'Université de Franche-Comté n ${ }^{\circ} 897$, p. 169-186

Duchet J.-L. \& Përnaska R., 1996, L'admiratif albanais, recherche d'un invariant sémantique, in Guentchéva (ed.), L'énonciation médiatisée, Paris, l'Information grammaticale, Louvain, Peeters, p. 31-46.

Franckel J.-J., 1989, Études de quelques marqueurs aspectuels du français, Genève, Droz.

Gevorkian G., 1994, The continuous present in armenian dialects, Annual of Armenian Linguistics 15, p. 43-62.

Guentchéva Z. (ed.), 1996, L'énonciation médiatisée, Paris, l'Information grammaticale, Louvain, Peeters.

Johanson L., 2000, Turkic indirectives, in Johanson L. and Utas B., Evidentials, Turkic, Iranian and Neighbouring Languages, Berlin/New-York, Mouton, p. 61-88.

Kasparian S., 1992, Analyse descriptive du parler multilingue d'Arméniens vivant à Paris, thèse de doctorat, Université de Paris III.

Kozintseva N., 1995, Modern Eastern Armenian, coll. Languages of the world / materials 22, München-Newcastle, LINCOM.

Landragin F., 2004, Saillance physique et saillance cognitive. CORELA Volume 2/Numéro 2. http://corela.edel.univ-poitiers.fr/index.php?id=603

Langacker R., 1991, Foundations of Cognitive Grammar, Stanford, Stanford University Press.

Langacker R., 1999, Grammar and Conceptualization, Berlin/New-York, Mouton.

Lazard G., 1956, Caractères distinctifs de la langue tadjik, Bulletin de la Société de Linguistique de Paris 52/1, p. 117-186.

Meydan M., 1996, Les emplois médiatifs de -miş en turc, in Guentchéva (ed.), L'énonciation médiatisée, Paris, l'Information grammaticale, Louvain, Peeters, p. 125-143.

Montaut A., 2006, Mirative Meanings as extensions of aorist in Hindi/Urdu, Yearbook or South Asian Linguistics, Amsterdam, Mouton, p. 71-86.

Milner J.-Cl., 1978, Le Système du réfléchi en latin, Langages 50, p. 73-86.

Aksu Koç A. \& Slobin D., 1986, A Psychological account of the development and use of evidentials in Turkish, in Chafe \& Nichols (eds), Evidentiality: The Linguistic Coding of Epistemology, Norwood, Abex Publ., p.159-167.

Tournadre N., 1996, Comparaison des systèmes médiatifs de quatre dialectes tibétains, in Guentchéva (ed.), L'énonciation médiatisée, Paris, l'Information grammaticale, Louvain, Peeters, p. 195-213.

Van der Auwera J. \& Plungian V.A., 1998, On modality's semantic map, Linguistic Typology 2, p. 79-124.

Vassilaki S. \& Tsamadou I., 1995, Aspects du grec moderne, Actes des sessions de linguistique et littérature à Aussois, LALIES 15, p. 7-70. 